RHIC-AP-19

INJECTION AND BEAM DUMPING FOR RHIC

(Recap of RHIC meeting dated 31 July 85)

J. Claus

BNL

November 4, 1985 
INJECTION AND BEAM DUMPING FOR RHIC

(Recap of RHIC meeting dated $31 \mathrm{July} 85$ )

Region available for this purpose: $D_{89}$ between $Q_{80}$ and $Q_{90}$ Characteristics of this space if $\beta *=3 \mathrm{~m}:$

Length (m)

Exit $Q_{80}$

$\alpha_{\mathrm{H}}$

$\beta_{H}(\mathbf{m})$

$\Delta \psi_{\mathrm{H}} / 2 \pi$

$n_{\mathrm{p}}(\mathrm{m})$

$\alpha_{\mathrm{V}}$

${ }^{B} V^{(m)}$

$\Delta \psi_{\mathrm{V}} / 2 \pi$

1.2397

$-0.0373$
21.6637

Entrance $Q_{90}$

0.3263

8.5815

0.15043

0.7281

$-1.6112$

49.4971

0.15565

We obtain the half aperture for the circulating beam by scaling from the high beta quads, which have $B=400 \mathrm{~m}$ and half aperture $\overline{\text { ha }}=60 \mathrm{~mm}$. Therefore: ha $\geq 60 \sqrt{B / 400} \mathrm{~mm}$. This rule neglects dispersive effects $\left(\eta_{p}\right)$

but this is acceptable.

Characteristics of injected beam:

$$
\mathrm{BP}=100 \mathrm{Tm} \text { (actually } \mathrm{B} \rho=90.018 \mathrm{Tm})
$$

Actual emittance $\varepsilon_{\mathrm{x}}=\varepsilon_{\mathrm{y}} \leq 0.83 \pi \times 10^{-6} \mathrm{rad}-\mathrm{m}$ for $2.5 \sigma$.

General Requirements.

The heavy ion beam to be injected comes in single bunches, one per AGS cycle. These bunches have to be deposited in empty buckets that are circulating in RHIC (so called box car stacking). The protons come in strings of 11 or 12 bunches, one string per AGS cycle. All bunches of a string have to be placed in waiting buckets, each bunch in its own bucket, in a single operation. This requires synchronism between the r.f. systems of AGS and RHIC at the time of 
beam transfer. The r.f. frequency in RHIC may be an integer multiple of that in the AGS. For various reasons that multiple was chosen to be 6 , so that, when injecting a string of proton bunches, only every sixth bucket will be filled. This choice yields a total of $h=342$ buckets around each ring of RHIC. Each bucket is $\lambda=\mathrm{c} / \mathrm{h}=3833.85 / 342=11.21 \mathrm{~m} \doteq 37.39 \mathrm{nsec}$ long. Imposing a ratio $\widetilde{\mathrm{B}}$ between bunch length $(2.50)$ and bucket length we have $2.50=\widetilde{\mathrm{B} \lambda}$. Choosing $\widetilde{\mathrm{B}} \leq 0.75$ in order to avoid making the synchrotron motion too non-linear we find for the r.m.s. bunch length

$$
\sigma=0.75 \times 11.21 / 2.5=3.363 \mathrm{~m} \doteq 11.22 \mathrm{nsec}
$$

For maximum luminosity it is desirable to fill each of the 342 available buckets. Our ability to do so is restricted by the switching time of the injection kicker. This magnet deflects the bunches that arrive from the beam transfer line between the AGS and one of RHIC's rings onto an orbit in that ring while it is on, it must be off when traversed by bunches of the circulating beam.

The switching time $\tau_{k s}$ is the time required by the kicker to change state, its turn-off time is not necessarily equal to its turn-on time. Bunches, or parts of bunches that are present inside the kicker's aperture while its state is being changed will be partially deflected, thus the tolerable switching time follows from the distance $\mathrm{N} \times \mathrm{C} / \mathrm{h}$ between the centers of successive filled buckets, the value of the bunching favor $\widetilde{B}$ and the velocity $\beta c$ :

$$
\sigma_{k s} \leq \frac{N-\stackrel{s}{b}}{h} * \frac{c}{B c}
$$

Substitution of $\rho=3833.85 \mathrm{~m}, \beta c=3 \times 10^{8} \mathrm{~m} / \mathrm{sec}, \mathrm{h}=342, \stackrel{\widetilde{R}}{\tilde{B}}=0.75$ yields

$$
\tau_{k s} \leq(M-0.75) \times 37.3 \mathrm{nsec}
$$

The evaluation of this expression in the interval $I \leq N \leq 6$ is given in Table I: 
Table I. Allowed switching times for injection kicker.

\begin{tabular}{crcc}
$\mathrm{N}$ & $\tau_{\mathrm{ks}}$ & $\mathrm{N}_{\mathrm{s}}$ & $\mathscr{L} / \mathscr{L}_{\mathrm{o}}$ \\
6 & 196 & 19 & 1 \\
5 & 159 & 22 & 1.15 \\
4 & 121 & 28 & 1.47 \\
3 & 84 & 38 & 2 \\
2 & 46 & 57 & 3 \\
1 & 9 & 114 & 6 \\
\hline
\end{tabular}

\section{Filling Patterns.}

It is easy to see that a lattice configuration with six equally spaced crossing points limits a bunch in one ring to interaction with only three particwular equal1y spaced bunches in the other. Those three bunches will exist only if the r.f. harmonic number is a multiple of three, interaction wil1 take place only if the bunches are properly phased relative to ring structure and each other. It follows that the bunches in each ring can be subdivided in sets of three, the bunches of each set being spaced equally around the ring, and that each set in a ring interacts exclusively with a particular set in the other. The collision rate in a particular insertion due to a matched pair of sets is $3 \times$ the revolution frequency, thus about $234.6 \mathrm{kHz}$, if there are $\mathrm{N}_{\mathrm{s}}$ sets in each ring the rate becomes $234: 6 \mathrm{~N}_{\mathrm{s}} \mathrm{kHz}$. The choice of harmonic number $\mathrm{h}=342$ implies $\mathrm{N}_{\mathrm{s}} \leq 342 / 3=114$, the $\mathrm{N}_{\mathrm{s}}$ value that can be realized depends on the switching times of the injection kicker. Table $I$ shows values for $\mathrm{N}_{S}$ and for the associated 1 uminosity multiplier $\mathrm{M}_{\mathrm{L}}=\mathscr{L} / \mathscr{L}_{\mathrm{o}}$. These results assume that the turn-on and turn-off times of the injection kicker are equal and as indicated. Only one of the two has to have the specified value, the other may be larger 
by a factor 2 or 3 without severe penalty since that would reduce the number of available buckets by only a small amount.

The bunches to be injected arrive from the AGS via a beam line that splits in two branches, one for each ring of RHIC. Each branch connects to the injection system proper near the downstream face of RHIC quadrupole $Q_{80}$ close to but outside its vacuum vessel. This places the centerline of the approaching beam at a distance of about $35 \mathrm{~cm}$ from the reference closed orbit since the vacuum vessel has an outside diameter of about $30 \mathrm{~cm}$. It is clearly desirable to choose that distance as small as possible, however, proportionally small changes bring only modest gains (or losses). A sequence of magnets, placed in the drift space between $Q_{80}$ and $Q_{90}$, guides the beam onto a suitable closed orbit in RHIC. For the moment we assume that orbit is the one which provides the largest acceptance for the circulating beam and also that it coincides with the reference closed orbit. The beam may approach either in the median plane of RHIC (horizontal injection) or above it and slope up or down (vertical injection). The last magnet of the sequence is the injection kicker which deflects the beam horizontally or vertically. We discuss three possible schemes.

The first one, which can be arranged for either vertical or horizontal injection, is attractive because of its conceptual and practical simplicity. It consist of only one magnet: the injection kicker. That kicker must be strong, however, e.g. $\int \mathrm{B} d l=3.5 \mathrm{Tm}, \theta_{k}=35 \mathrm{mrad}, \mathrm{L}_{k}=20 \mathrm{~m}$, so that $i t$ is very expensive and not very suitable for small switching times.

The second scheme is similar to the injection system planned for CBA: the beam passes through a $1.5 \mathrm{~T} 4.2 \mathrm{~m}$ long septum magnet, a $4 \mathrm{~m}$ 1 ong Lambertson magnet whose field is graded from $1.5 \mathrm{~T}$ near its entrance to $0.5 \mathrm{~T}$ near its exit and a $3 \mathrm{~m}$ long $0.34 \mathrm{Tm}$ vertically deflecting kicker. The constraints imposed by 
geometry and aperture are much more severe in RHIn, than they were in CBA, both the septum magnets and the kicker have to be considerably stronger. The margin available for septum magnet development is very small because of iron saturation, and the slack has to be absorbed by increasing the strength of the kicker. The numbers quoted in Table II are based on optimistic assumptions for the septum magnets and optimized for minimum kicker strength.

The third scheme is based on conventional median plane injection. Two horizonally deflecting septum magnets guide the beam towards a horizontally deflecting kicker as shown in the layout in Fig. 1.

This scheme requires the weakest kicker of the three and has a relatively simple beam trajectory, all in the median plane. It also offers the best prospects for further development towards minimum kicker requirements. It needs serious work however, particularly on the septum magnets. Unless these magnets are made superconductive they have to be pulsed for dissipation reasons. Half sinusoidal pulses with a base width of $s 1 \mathrm{msec}$ would be adequate. They would require either laminated iron or ferrite cores and would have to be bakeable in situ if they are located inside RHIC's u1tra high vacumm beam tube. It has been shown that ferrite cores can be baked and are acceptable with regard to vacuum. These ferrites saturate at $0.3 \mathrm{~T}$, while we need at least $0.53 \mathrm{~T}$ over a length of $9 \mathrm{~m}$. Since there is no prospect of achieving this we introduced two septum magnets, the first one for $1.6 \mathrm{~T}$ with a laminated core separated from the beam vacuum by a bakeable, thin walled, stainless steel beam pipe, the second one a $0.3 \mathrm{~T}$ ferrite magnet with a $1 \mathrm{~mm}$ thick current septum inside the vacuum. Other configurations are clearly possible, e.g. ones with a vacuum pipe through both magnets . 


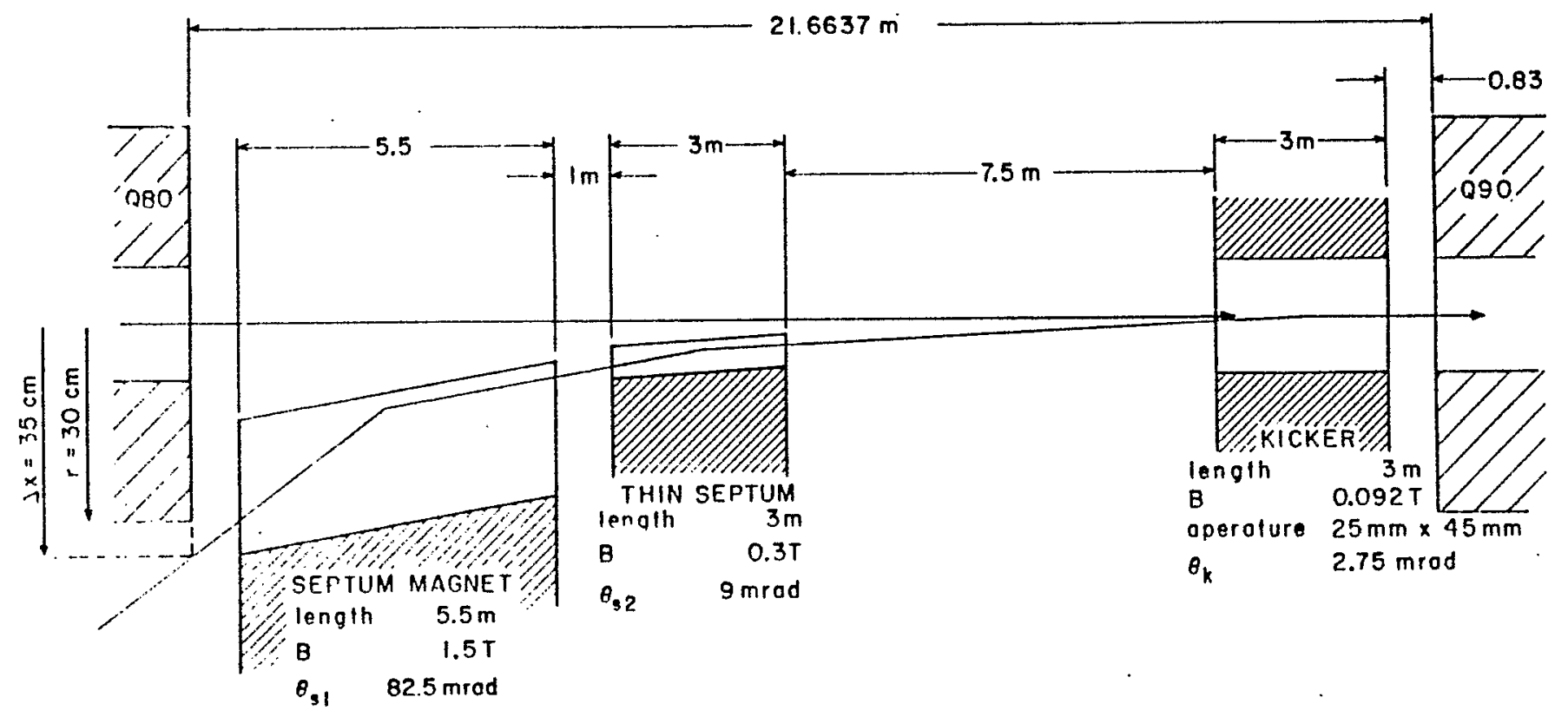

Figure 1. Injection component layout. 


\section{Tolerances.}

There are many tolerances to be satisfied. We consider only the ones that might change from injection to injection, i.e. field inaccuracies in septum magnets and injection kicker. The tolerances follow from the requirements that the error in deflection angle, which causes coherent betatron motion of the injected bunch, may not increase the effective emittance of the circulating beam by a predetermined amount. Taking for this amount e.g. $4 \%$ of the nominal emittance of $0.8 \times 10^{-6} \pi \mathrm{rad}-\mathrm{m}$ we find for the example of horizontal injection given

$$
\begin{aligned}
& \left(\frac{\Delta B}{B}\right)_{s_{1}} \leq 1.7 \times 10^{-4} \\
& \left(\frac{\Delta B}{B}\right)_{s_{1}} \leq 3.4 \times 10^{-3} \\
& \left(\frac{\Delta B}{B}\right)_{k} \leq 1.3 \times 10^{-2}
\end{aligned}
$$

These magnets are only the last three of a chain that begins with the magnets for the extraction closed orbit bump in the AGS and which contains its extraction kicker. If we allow only six elements equal uncorrelated proportional errors it follows that for $10 \%$ net error one can allow not more then $0.1 / \sqrt{ } 6=$ 0.04 per element.

\section{Kicker Considerations.}

A kicker may be characterized by its strength $\left(\int_{B} d l\right)$, aperture $(w \times g)$, length $(l)$ and speed $\tau_{\mathrm{ks}}$. Assuming a window frame configuration and single turn excitation we have approximately for the excitation current I:

$$
I=\frac{g}{\mu_{0} l} \int \dot{B} d l=\frac{g}{\mu_{0}^{l}} \theta_{k}(B \rho),
$$

for the inductance $L$ : 


$$
\mathrm{L}=\mu_{\mathrm{o}} \frac{\mathrm{wl}}{\mathrm{g}}
$$

for the stored energy:

$$
E_{m}=\frac{1}{2} L I^{2}=\frac{1}{2} \frac{g w}{\mu_{o}^{l}}\left(\theta_{k} B \rho\right)^{2}
$$

and for the voltage:

$$
V=L \frac{d i}{d t}=L \frac{I}{\tau_{k s}}=w \theta_{k}(B \rho) / \tau_{k s}
$$

The function of the injection kicker is to generate displacement within a drift space of limited length. Calling the displacement $d$ and the driftspace length $s$ we have

$$
\theta_{k}=d /(s-1 / 2 l)
$$

thus

$$
E_{m}=\frac{1}{2} \frac{g w}{\mu_{0} l}\left(B \rho \frac{d}{s}\right)^{2} \frac{1}{\lambda\left(1-\frac{1}{2} \lambda\right)^{2}}
$$

where $\lambda=\ell / s<1$

This function has a minimum for $\lambda=2 / 3$, i.e. for a kicker length equal to $2 / 3$ of the available space, given by

$$
\mathrm{E}=\frac{1}{2} \frac{g \mathrm{w}}{\mu_{\mathrm{o}} l}\left(\mathrm{~B} \rho \frac{\mathrm{d}}{\mathrm{s}}\right)^{2} \times 3.375
$$

An optimally positioned kicker half as long would require

$$
E_{m}=1 \cdot 28 E_{m}^{v} .
$$


It is assumed in this estimate that the kicker aperture does not change along its length. Aperture width and height have then to be larger than the largest expected beam width and height anywhere inside the magnet and increase with length because of the behaviour of the $B$ functions in a drift space. This results in our case in a width that increases proportionally with length. Another factor in that the beam displacement due to the kicker action increases with length for fixed d and extra aperture width may have to be provided to accommodate it. There are thus several factors that enter in the minimization of the stored energy.

Typical numbers of RHIC injection kickers are given in Table II.

Table II. Typical kicker parameters.

\begin{tabular}{|c|c|c|}
\hline Mode of injection & Horizonta1 & Vertical. \\
\hline Aperture $(w \times g)(m m \times m m)$ & $25 \times 45$ & $45 \times 25$ \\
\hline Length $\quad(l)(\mathrm{m})$ & 3 & 3 \\
\hline$B(T)$ & 0.0916 & 0.113 \\
\hline$I(A)$ & 3280 & 2250 \\
\hline $\mathrm{L}(\mu \mathrm{H})$ & 2.1 & 6.8 \\
\hline $\mathrm{v}(\mathrm{kV})$ & 46 & 102 \\
\hline $\mathrm{E}_{\mathrm{m}}(\mathrm{J})$ & 11.26 & 17.14 \\
\hline $\int \mathrm{B}$ dl $(\mathrm{Tm})$ & 0.2748 & 0.339 \\
\hline $\mathrm{B} \rho(\mathrm{Tm})$ & 1.00 & 100 \\
\hline$\theta_{k}(\operatorname{mrad})$ & 2.75 & 3.4 \\
\hline
\end{tabular}




\section{Improvements.}

The choice of the reference closed orbit as injection closed orbit is conceptually convenient. It provides a large aperture for the circulating beam but requires a strong, ful1 aperture kicker. That aperture is necessary to accommodate the growth in beam emittance due to intrabeam scattering and is not fully used during the injection, except perhaps for beam manipulation. Since the emittance growth occurs on a time scale that is large compared to the injection interval it is acceptable to choose a different closed orbit, closer to the septum of the critical second septum magnet, provided that orbit is moved towards the reference orbit as necessary to compensate for the increasing beam size. The smaller distance between septum and orbit allows a smaller kicker deflection angle. It is also acceptable to use a kicker with a smaller aperture, provided that it is moved out of the way before it begins to intercept circulating beam. This can be done because there is ample time (minutes). Reductions in deflection and gap width result in a reduced voltage for a fixed switching time $\tau_{k s}$ or in a decreased switching time for fixed voltage. We estimated the gain for two cases. In one we adhere to the rule that the available aperture should provide for at least $6\left(\sigma_{\beta}+\sigma_{E}\right)$ a11 around the ring, in the second we assume that $2.5\left(\sigma_{\beta}+\sigma_{E}\right)$ is acceptable in the vicinity of the second septum magnet and in the kicker aperture during the injection period. We express the results in terms of the kicker requirements for injection onto the reference closed orbit. They are given in Table III. We used $\beta=32.5 \mathrm{~m}, \eta_{p}=1 \mathrm{~m}$ for the focussing parameters at the exit of the second septum magnet, assumed a septum thickness of $1.5 \mathrm{~mm}$, and allowed for $2.5\left(\sigma_{B}+\sigma_{E}\right)$ in the beam to be injected at its exit. 
It is evident that acceptance of a minor amount of beam scraping during injection should reduce the cost of kicker and kicker energy storage and permits a five fold decrease in switching time for the same kicker voltage. It will be very difficult to keep the kicker outside the beam vacuum in this approach.

Table III. Relative changes in kicker parameters.

\begin{tabular}{lrc}
\hline & $6\left(\sigma_{B}+\sigma_{E}\right)$ & $2.5\left(\sigma_{B}+\sigma_{E}\right)$ \\
$A_{k} / \theta_{\mathrm{kro}}$ & 0.90 & 0.54 \\
gapw $/ g a p w_{\text {ro }}$ & 0.86 & 0.36 \\
gaph/gaph ${ }_{\text {ro }}$ & 0.86 & 0.36 \\
$\mathrm{E}_{\mathrm{m}} / \mathrm{E}_{\mathrm{mro}}$ & 0.67 & 0.07 \\
$\left(\mathrm{~V} / \mathrm{V}_{\mathrm{ro}}\right)_{\mathrm{ks}}$ & 0.76 & 0.20 \\
\hline
\end{tabular}

Beam Dumping

The energy stored in the circulating beam in one of the RHIC rings is nomina11y of the order of $300 \mathrm{~kJ}$, only $1 / 130$ of what was expected in CBA. Improvements in the source, i.e. Tandem, Booster and AGS and reductions in the switching times of the injection kicker could increase it by a factor 20 to 6 MJ . This energy must be dissipated, without causing damage or driving the superconducting magnets normal, whenever the beam has to be disposed of at the end of a run or because of some accident or malfunction. The energy stored in CERN's ISR beams reached 4 M.J and was dissipated routinely in an internal dump target. It is, therefore, reasonable to plan the beam dumping system of RHIC also around an internal dump. It is prudent however to allow for extraction and an external beam dump in case this should prove desirable or necessary. The beam dump system consists then of a dump kicker and a dump target; this target 
to be replaceable by a first extraction septum magnet eventually. The extraction kickers have to be strong enough to move a beam from anywhere in the aperture onto the dump target. Ideally their switching time should be similar to that of the injection kickers and the initiation of extraction should be synchronized to the bunch structure of the circulating beam to prevent uncontrolled loss of beam somewhere along the ring circumference. The kicker aperture must be sufficiently large to prevent any obstruction of the circulating beam and the shunt impedance it presents to the circulating beam should be controlled to minimize enhancement and to avoid introduction of instabilities. Inspection of the current RHCI lattice suggests vertically deflecting kickers around one of the $Q_{2 I}$ insertion quadrupoles and a beam dump or extraction septum magnet between $Q_{5 I}$ and $Q_{6 I}$, as indicated in Fig. 2. The vertical deflection allows us to take advantage of the fact that the beam's median plane is much less uncertain than its behavior in that plane. In the case of extraction, the beam would pass through the aperture of $Q_{4 I}$ on its way out. Another possibility is to have the septum magnet direct the beam, via the aperture of $Q_{4 I}$, to a very elaborate dump target in the $38 \mathrm{~m}$ long drift space between $Q_{4 I}$ and $Q_{9 I}$. Various arrangements were tried and some results are shown in Table IV. The cases shown there have respectively a kicker'K1 only, a kicker $\mathrm{K} 2$, kicker $\mathrm{K} 1$ and kicker $\mathrm{K} 2$, both with the same $B$ field and kickers $K 1, K 2$ and $K 3$, now all three with the same $B$ field. $\mathrm{K} 2$ and $\mathrm{K} 3$ are restricted in their lengths on both sides by lattice magnets, $\mathrm{KI}$ only at its down stream end. Whether the cross sections of the various kickers should be identical or adjusted to match the dimensions of the circulating beam is a complex economic question we do not discuss. It may be seen that in all four cases $\Sigma \int \mathrm{B} d \mathrm{~d}, \sim 0.7 \mathrm{Tm}$, and that the $\mathrm{B}$ field decreases with increasing kicker length. The table gives the minimum free aperture ( $w \times h)$ required in 


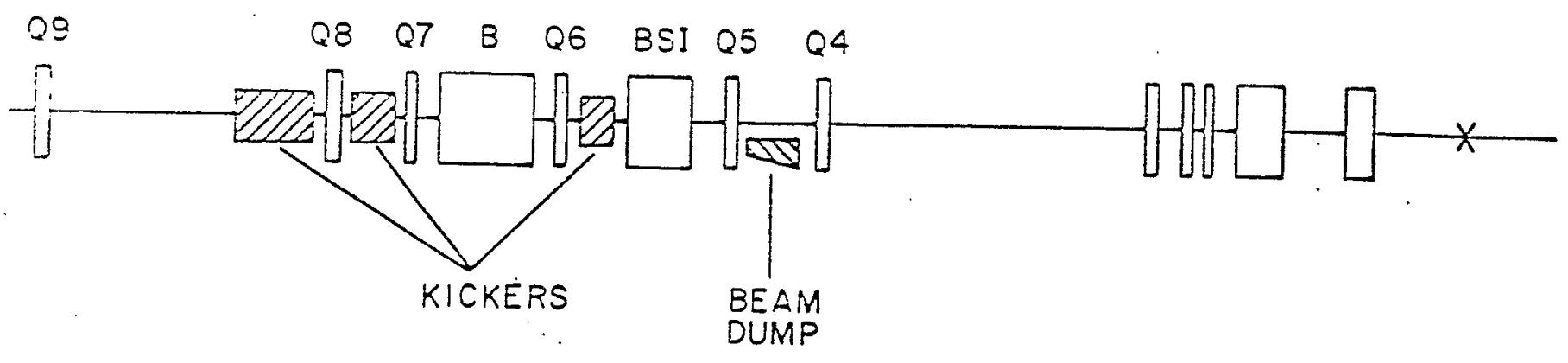

Figure 2. 


\footnotetext{
each kicker and the energy in the kicker fields per meter kicker length associated with them.

If we use $K_{2}$ exclusively it will have to be rather strong ( $B=0.23 \mathrm{~T}$ for particles with the maximum magnetic rigidity $\mathrm{B} \rho=839.5 \mathrm{Tm}$ ). This will make it difficult to provide it with acceptably small switching times. Having only $\mathrm{Kl}$ releaves this problem because $\mathrm{Kl}$ can be given nearly arbitrary length, but doing so imposes larger aperture requirements in BS1, Q5I and BS2. 'Using both

$\mathrm{K} 1$ and $\mathrm{K} 2$ improves the situation in this respect, but not enough. This was the reason for adding $\mathrm{K} 3$. It may be seen that there is an aperture problem, at least at $Q_{5} \mathrm{I}$, in all four cases.
} 
Table. Beam dumping

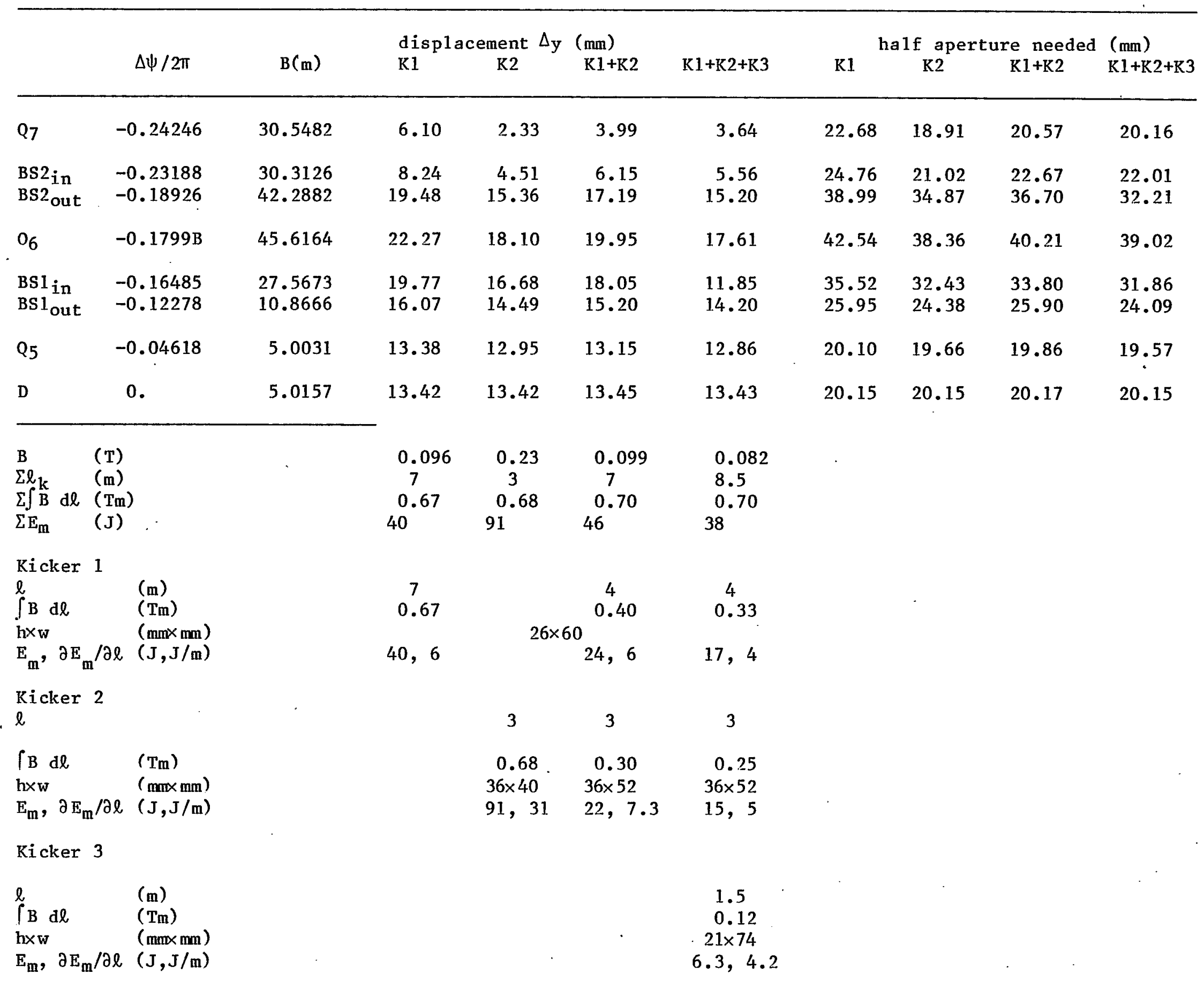

